\title{
Les Cooperatives Et La Gestion De La Relation Client (CRM) : Quelles Demarches De Perfectionnement ?
}

\author{
Khadija Aberji \\ Doctorante au LARCEPEM, Faculté Des Sciences Juridiques, \\ Économiques Et Sociales Souissi, Université Mohammed V, Maroc
}

Doi:10.19044/esj.2019.v15n10p205 URL:http://dx.doi.org/10.19044/esj.2019.v15n10p205

\section{Résumé}

L'environnement des coopératives au Maroc est en mouvement permanent à l'heure actuelle, chose qui suppose la mise en place de nouvelles stratégies pour s'adapter à un tel environnement et relever le défi de la performance. Ce mouvement touche tous les périmètres de la coopérative, notamment sa relation avec ses clients.

L'objectif de ce papier est de montrer à travers la littérature et le terrain que la gestion de la relation avec les clients est un enjeu stratégique pour la coopérative, dans la mesure où elle impact directement sa performance, et de proposer des mesures susceptibles d'améliorer cette dernière. En effet, nous avons pu constater que les coopératives au Maroc ne sont suffisamment pas conscientes de l'importance de la CRM pour leur performance, et qu'elles sont dominées par un style managérial traditionnel qui néglige le digital et les fortes transformations sociétales.

Mots Clés : Coopératives, compétitivité, performance relationnelle, la gestion de la relation clients. 


\title{
Cooperatives and Customers Relationship Management: What Development Measures?
}

\author{
Khadija Aberji \\ Doctorante au LARCEPEM, Faculté Des Sciences Juridiques, \\ Économiques Et Sociales Souissi, Université Mohammed V, Maroc
}

\begin{abstract}
The environment of cooperatives in morocco is in constant motion at the moment; which supposes the implementation of new strategies to adapt to such environment and, to meet the challenge of performance. This movement affects all the perimeters of the cooperative, particularly its relationship with its customers.

The purpose of this paper is to demonstrate through literature and the ground that managing the relationship with customers is a strategic issue for the cooperative as it directly affects its performance, as well as proposing reliable measures to ameliorate this latter. Hence, we have been able to witness that the cooperatives in Morocco are not sufficiently conscious of the importance of the CRM for their performance, and that they are dominated by an out of date managerial style, which neglect the new technologies and the social transformations.
\end{abstract}

Keywords: Cooperatives, Competitiveness, relation performance, Customer relationship management.

\section{Introduction :}

Les fortes évolutions en cours depuis la révolution technologique ont contribué à déstabiliser l'environnement et l'organisation interne voire le quotidien des entreprises sociales particulièrement les coopératives. De profondes transformations sont enregistrées sous l'impact des nouvelles technologies de l'information et de la communication (NTIC), et du changement à une grande échelle des modes de vie, de production et de consommation. Ces transformations ont une influence sur tous les périmètres de la coopérative, notamment ses relations avec ses clients. Dans ce contexte, nul ne peut nier que les relations avec les clients ont vécu des mutations profondes au fil des années, et que le perfectionnement de ces relations est un véritable défi devant les coopératives; un défi qu'elles doivent considérer comme une des priorités majeures pour rester compétitives et performantes. 
Aujourd'hui, face aux sérieux défis soulevés par la compétitivité et la performance, les coopératives s'interrogent sur les moyens sur lesquels elles peuvent se focaliser pour mettre en valeur leur relation avec leurs clients, en tant qu'une vraie richesse et une partie incontournable dans le processus de création de la valeur ajoutée. Etant dans l'ère du digital, les clients ne sont plus de simples consommateurs (consommateurs aveugles), mais plutôt des parties bien informées et plus exigeantes en termes de qualité du produit et de maturité de la coopérative.

En effet, comprendre et analyser ce nouveau comportement des clients «contemporains" constitue pour la coopérative un pas réussi vers leur satisfaction parfaite et leur fidélisation. Dans ce sens, la coopérative ne doit plus constituer uniquement une entité qui cherche à réaliser une richesse sociale puis économique, mais aussi à créer des liens sociaux solides et à long terme, notamment avec sa clientèle.

La création de liens de telle nature, qu'on peut qualifier de performance relationnelle, suppose pour la coopérative d'aller au-delà des pratiques traditionnelles, vers la mise en scène des expériences particulières et mémorables. Pour ce faire, l'expérience doit faire appel aux émotions (Laurence Body, 2011), plus le sentiment est formidable, plus l'expérience est marquante, et plus la concurrence est faible (Pine et Gilmore, 1999).

Notre réflexion part du constat que les recherches qui suggèrent de véritables mesures à l'attention des coopératives pour perfectionner leur relation avec leurs clients sont rares. A cet effet, nous proposons de montrer que la prise en considération par les coopératives de l'évolution continue des attentes de leurs clients suite à l'évolution du digital a un impact actif et direct sur leur compétitivité, ainsi que la mise en place de nouvelles démarches pour la fidélisation de ces clients pourrait apporter une réponse potentille aux défis soulevés par la compétitivité et la performance.

En d'autres termes, nous cherchons à comprendre en quel sens le renforcement des liens avec les clients pourraient contribuer au renforcement de la compétitivité et la performance des coopératives, en proposant certaines mesures susceptibles de réussir cette équation.

Cet article s'articule autour de deux parties. Dans la première partie nous présentons dans un cadre conceptuel les liens entre les coopératives et la gestion de la relation client. Dans la deuxième partie, nous exposons les résultats de l'enquête menée auprès d'un échantillon de coopératives ainsi que les différentes démarches qui permettent aux coopératives de fidéliser leurs clients et se rendre en effet compétitives. 


\section{LES COOPERATIVES ET LA GESTION DE LA RELATION CLIENTS (CRM) : CADRE THEORIQUE}

Ce cadre théorique a pour objectif de montrer dans quelle mesure une performance relationnelle avec les clients assure une forte compétitivité aux coopératives.

\subsection{LES COOPERATIVES AU MAROC: BREF ETAT DES LIEUX}

«Les coopératives construisent un monde meilleur»: sous cette devise que les nations unies ont reconnu en 2012 via leur assemblée générale l'importance des coopératives pour le développement socioéconomique dans un pays donné. Etant un acteur pivot de l'économie sociale et solidaire, les coopératives peuvent être considérées comme un groupement de personnes physiques ou morales qui conviennent de se réunir pour créer une entreprise, dans le but d'améliorer les conditions de vie de leurs adhérents, et de contribuer à la réduction de la pauvreté, de l'exclusion sociale et du chômage, essentiellement dans les zones et régions où sont installées.

En ce sens, les coopératives ont un rôle très important pour le développement socioéconomique d'un pays donné, du fait qu'elles ont la capacité de contribuer activement à l'amélioration des conditions de vie de leurs adhérents à travers des activités économiques génératrices de revenus, créatrices d'emplois et de richesses. En effet, elles conjuguent utilité sociale et efficacité économique, en favorisant ainsi l'émergence d'une nouvelle forme d'entrepreneuriat qui priorise le social et oppose la logique capitaliste qui ne cherche que le profit et l'accumulation des richesses.

De même, les coopératives ont certaines spécificités économiques et managériales qui les distinguent des autres formes d'entreprises, notamment: autonomie et indépendance, adhésion volontaire et ouverte à tous, égalité entre adhérents (pouvoir exercé démocratiquement), bénéfices et risques équitablement partagés, engagement fort envers la communauté.

Ces entités qui ont connu une évolution remarquable dans plusieurs pays, particulièrement en Europe, suscitent un intérêt croissant ces dernières années au Maroc, vu qu'elles sont reconnues comme étant une solution innovante aux problèmes majeurs de la société (pauvreté grimpante, chômage amplifié, exclusion sociale, disparités territoriales, analphabétisme...). Dans ce sens, le gouvernement marocain a mis en place plusieurs mesures (appui institutionnel et fiscal notamment) pour favoriser la création des coopératives et renforcer leur contribution au développement socioéconomique du pays; d'où l'évolution continue du nombre des coopératives créées. 
Figure $\mathrm{N}^{\circ} 1$ : Evolution du nombre des coopératives au Maroc entre 2010 et 2015

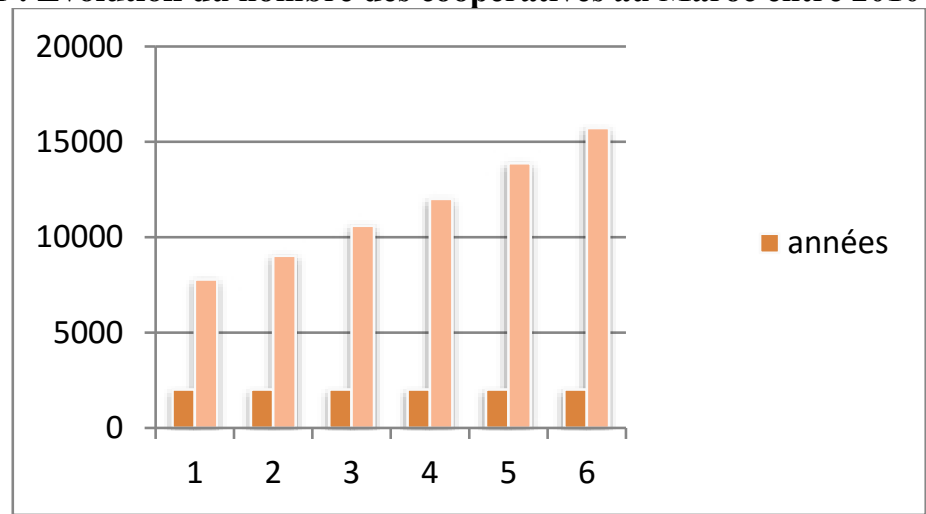

Source : ODCO, annuaire statistique des coopératives et leurs unions au Maroc pour l'année 2015

Figure $\mathrm{N}^{\circ} 2$ : évolution des coopératives selon le secteur d'activité.

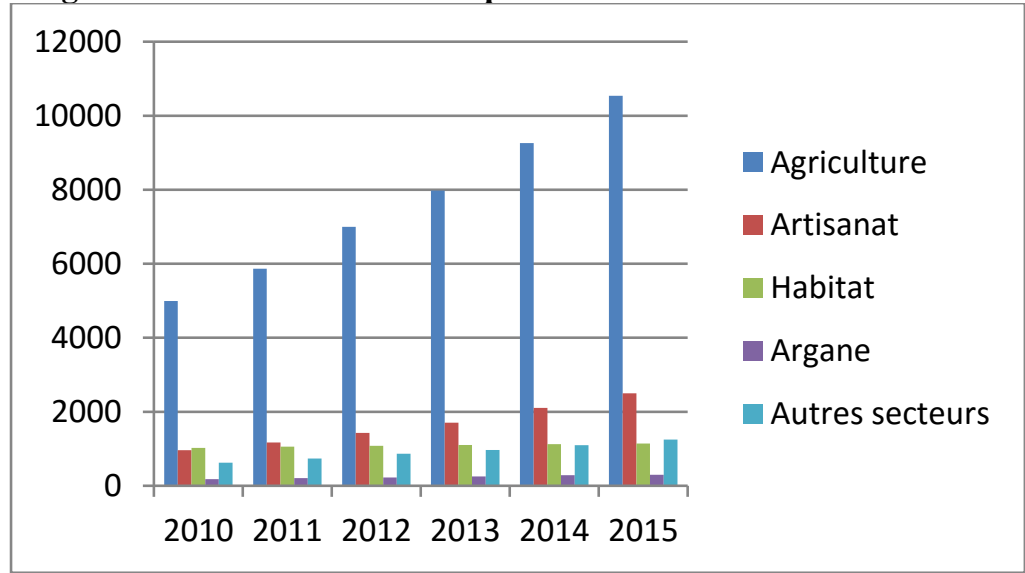

Source : ODCO, annuaire statistique des coopératives et leurs unions au Maroc pour l'année 2015

La croissance des coopératives en nombre ne s'est pas traduite au niveau de la richesse nationale créée. En effet, la participation des coopératives dans le PIB du pays se limite à 1,6\% selon les statistiques de 2016 selon Abdelkrim AZENFAR le directeur de l'ODCO. Cet état des choses est expliqué essentiellement par la pauvreté des mesures prises par l'Etat en termes d'innovation et leur déconnexion des transformations vécues par la société contemporaine ; ainsi que la non prise en compte par les coopératives des mouvements de leur environnement et de l'impact des fortes évolutions sociétales vécues, c'est la raison pour laquelle un grand nombre de coopératives s'orientent vers des secteurs d'activité traditionnels, notamment l'agriculture et recourent dans ces secteurs à des techniques classiques pour la production et la commercialisation, alors que les secteurs intégrant les nouvelles activités qui pourraient répondre aux nouveaux besoins de la société 
(secteur des services par exemple) n'attirent pas jusqu'à ce jour une grande partie du réseau coopératif au Maroc. Ceci peut être la résultante du manque voire l'absence de l'intervention des parties concernées (notamment le ministère de tutelle et l'OCDO) en matière d'orientation, de sensibilisation et bien évidement de formation au profit des membres des coopératives dès les premières étapes de constitution de la coopérative ; avec tout ce que cela pourrait engendrer comme conséquences, particulièrement le lancement d'une coopérative sur un marché mal connu, et dans un environnement mal maitrisé.

Dans cette perspective, la favorisation par les coopératives d'une « vocation produit/prix » ne peut plus être à elle seule une stratégie efficace pour se développer et s'imposer sur un marché en mouvement permanent et caractérisé par une concurrence acharnée.

De ce fait, les coopératives d'aujourd'hui doivent avoir conscience de l'impératif de s'adapter aux nouvelles exigences de la compétitivité, notamment une excellente gestion de la relation avec leurs clients, celle-ci suppose entre autres : l'utilisation des réseaux sociaux pour établir des liens sociaux forts et particuliers avec les clients, l'utilisation des applications mobiles pour fournir des services, et la favorisation d'une culture du digital au sein des coopératives.

Ainsi, cette relation placée au centre des préoccupations des entreprises de toutes natures a connu une transformation radicale à l'heure du digital.

\subsection{LA GESTION DE LA RELATION CLIENT : UN ENJEU STRATEGIQUE POUR LES COOPERATIVES}

Sans doute, le client est un acteur stratégique pour toute entreprise, de toute forme et de tout statut, y compris les coopératives; cet acteur est devenu aujourd'hui bien informé et de plus en plus exigeant suite aux nouvelles technologies ; ces dernières ont également fait évoluer les comportements des clients ; leurs goûts changent de manière continue, ainsi que leurs attentes qui évoluent vers la recherche de la particularité de la relation avec l'entreprise. De ce fait, donner un sens singulier voire unique à cette relation est possible grâce aux nouvelles démarches de fidélisation notamment la CRM (Customer Relationship Management).

La gestion de la relation client, connue souvent sous son acronyme anglais CRM est l'ensemble des techniques et mesures qui permettent à l'entreprise l'établissement d'une relation durable et marquante avec ses clients. Plus encore, c'est une stratégie qui vise la performance relationnelle avec les clients, en automatisant les différents aspects de la relation client. La mise en place de cette stratégie requiert 1'exploitation intelligente de la technologie digitale.

A vrai dire, placer la technologie au cœur de la stratégie de la coopérative est un outil intéressant qui lui permet de mieux connaitre ses clients, de 
découvrir leurs attentes et de comprendre voire anticiper leurs demandes (big data). En ce sens, se référer à la CRM permettra à la coopérative de segmenter ses clients afin de leur proposer des services et/ou biens adaptés à leurs besoins, chose qui servira par la suite à leur fidélisation, en privilégiant ainsi l'idée selon laquelle conquérir un prospect coûte beaucoup plus cher que fidéliser un client.

Dans cette logique, les coopératives se trouvent aujourd'hui devant une clientèle connectée, bien informée, et fait souvent recours aux techniques de l'information et de la communication, notamment l'internet avant de prendre une décision de consommation. A ce niveau, nous constatons qu'il est utile de mentionner que le pourcentage d'internautes (pages web, les réseaux sociaux...etc) est en forte évolution partout dans le monde : 3,81 milliards d'internautes, soit $51 \%$ de la population mondiale (T. Coeffé, 2017). Au Maroc, les statistiques de l'ANRT parlent de 60\% d'internautes en 2017 soit 19 millions, avec $73 \%$ des personnes âgées de plus de cinq ans sont équipées d'un smartphone. Cela signifie que l'accès à l'information est devenu de plus en plus facile et à la portée de tout le monde et à tout moment, d'où l'idée de prendre en considération cette nouvelle donnée s'impose sur la coopérative, notamment pour se démarquer et gagner une place sur un marché en forte concurrence et évolution.

Par conséquent, les coopératives comme toutes les autres entreprises doivent désormais prendre en compte le potentiel que leur offre les nouvelles technologies et les objets connectés (les Smartphones bien évidemment) ; on parle surtout d'impliquer les clients comme toutes les autres parties prenantes dans le processus de création et du lancement d'un nouveau produit, ou du renouvellement d'un produit déjà existant; cette implication pourrait permettre à la coopérative de graver un souvenir inoubliable sur la mémoire de ses clients, et donner l'impression à chacun que le produit est fabriqué exclusivement pour lui-même, c'est « l'expérience client » que nous visons.

L'expérience client est une nouvelle approche de différenciation et de personnalisation axée sur les émotions et vise l'accompagnement du client, avant, pendant et après l'achat (L.Body, C.Tallec, 2015) ; c'est une phase audelà d'une simple satisfaction du client, et d'une relation de qualité, vers des émotions extraordinaires et un moment du plaisir incroyable. De ce fait, un client enchanté suite à une opération d'achat, revient souvent pour acheter davantage, en recherchant le même degré de satisfaction et le même plaisir. Cet enchainement peut être illustré d'après la pyramide ci-dessous : 
Figure $\mathrm{N}^{\circ} 2$ : les niveaux de l'expérience client

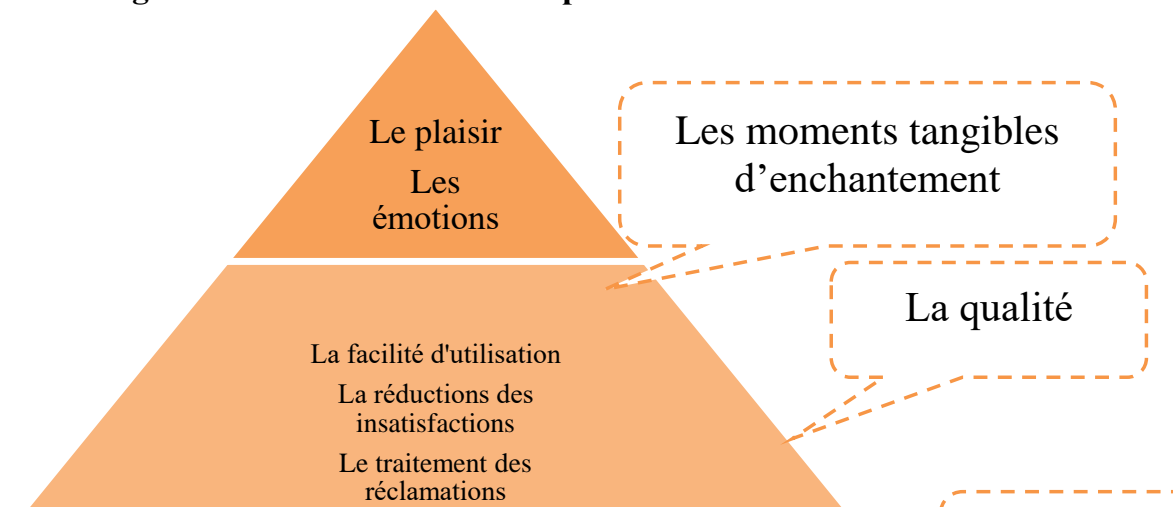

Proposition de valeur centrale

La satisfaction des besoins

Source : L. Body \& C. Tallec (2015), “L'expérience client”, EYROLLES,

Répondre aux exigences des clients en termes d'expérience engendre de profondes transformations dans l'organisation interne de la coopérative, notamment se débarrasser des démarches obsolètes et traditionnelles du management, et abandonner les analyses simplistes des faits et des changements de l'environnement. En effet, il est primordial de connaitre ses clients afin de savoir comment les satisfaire et leur permettre de vivre une expérience particulière; leur connaissance permettra aussi de réussir à accompagner le changement de leurs comportements et créer des liens humains forts, durables et mémorables. La prise en considération par les coopératives de ces facteurs assure leur survie et compétitivité à condition de suivre une méthodologie structurée pour créer une conjoncture favorable à son déploiement.

\section{COOPERATIVES MAROCAINES ET LA RELATION AVEC LES CLIENTS : REALITE ET DEMARCHES DE PERFECTIONNEMENT}

Dans cette partie de cet article, loin de la théorie nous exposons une image réelle de la relation des coopératives au Maroc avec leurs clients et l'ensemble des facteurs qui affectent cette relation, et impactent en effet leur compétitivité. Nous cherchons à proposer par la suite certaines démarches qui pourraient permettre aux coopératives marocaines de se rendre compétitives. 


\subsection{LA RELATION DES COOPERATIVES AU MAROC AVEC LEURS CLIENTS : UN APPERCU DE LA REALITE}

Comme déjà indiqué, le nombre de coopératives créées au Maroc est en forte évolution d'une année à l'autre sur l'ensemble du territoire national, notamment après le lancement de l'INDH en 2005. En revanche, cet accroissement en nombre n'est pas accompagné par une croissance sur d'autres volets, particulièrement le volet managérial au sein des coopératives, et encore plus particulièrement le management des relations.

Ce constat, suppose de faire une étude sur le terrain pour comprendre la réalité des choses, et avoir une vision plus claire sur la nature de la relation des coopératives avec leur clientèle, et comment est-elle appréciée et prise en compte en leur quotidien.

L'étude menée par nos soins à travers des entretiens semi-directifs avec les représentants des coopératives, a été faite sur un échantillon de 81 coopératives dans la région de Marrakech-Safi, de tailles petites et moyennes, et opérantes dans différents secteurs : agricole, artisanat, argane, alimentaire, et plantes médicinales et aromatiques. Ces coopératives, opèrent essentiellement dans des communes rurales de la région. Par la suite nous avons généralisé les résultats obtenus.

Notre échantillon est choisi au hasard -dans le sens scientifique et mathématique du terme- selon la technique aléatoire dirigée (J.L.Loubet del Bayle, 2000), en se référant à une liste des coopératives installées à la région de Marrakech-Safi, récupérée auprès de l'office du développement de la coopération. Ainsi, le choix de la région de Marrakech-Safi est justifié par trois principales raisons à savoir : * Marrakech-Safi est la deuxième région la plus pauvre du Maroc, avec un taux de $11.3 \%$ (rapport officiel), * un réseau coopératif très important, * raisons de mobilité.

Plus encore, pour étudier notre échantillon et répondre en effet à notre objectif nous avons opté pour une démarche qualitative à caractère exploratoire, basée sur des entretiens semi-directifs menés à l'aide d'un guide d'entretien, utilisé justement pour mieux structurer nos entretiens et obtenir des éléments des réponses pertinents. Pour cela, nous avons fait recours à l'analyse de contenu comme méthodologie la plus fréquemment utilisée pour analyser les données, propos et informations recueillies auprès des interviewés lors des entretiens. Cette analyse nous a permet de comprendre, de décrire et d'évaluer la situation managériale (management des relations notamment) des petites et moyennes coopératives particulièrement à la région Marrakech-Safi et au Maroc en général, et aussi de découvrir les facteurs et les comportements de leur succès et d'échec. Pour obtenir les chiffres annoncés (les pourcentages évidemment) nous avons fait recours au fameux logiciel Excel.

D'après l'exploitation des réponses de nos interlocuteurs, une première observation « urgente » s'exige, les coopératives au Maroc sont dominées par 
une gestion classique à tous les niveaux : production, commercialisation, relation client, planification, veille stratégique...Etc. En effet, aucune stratégie n'est définie en amont de manière consciente et précise.

Bien qu'elles sont créées au début dans le but de soutenir une population qui vit dans la pauvreté et la précarité, une population marginalisée, suite à la domination du capitalisme « sauvage »; les efforts du ministère de tutelle ont été centrés sur l'élargissement du réseau des coopératives plutôt que leur modernisation et la préparation d'un contexte favorable pour s'adapter aux grands mouvements de l'environnement, et aux changements des modes de vie, de production et de consommation, ainsi que le renforcement de leur contribution au développement socioéconomique.

D'après les entretiens menés avec les représentants des coopératives, plus de $80 \%$ des coopératives produisent en utilisant des techniques traditionnelles ou moins sophistiquées (parfois à la main) malgré qu'elles existent pendant des années. De ce fait, très peu de coopératives intègrent la technologie dans leur quotidien ou dans le processus de création, de fabrication, de production et même de commercialisation de leurs produits, cela peut être expliqué par le fait que les coopératives interrogées ne réalisent pas des gains importants de façon à investir une partie de ces gains dans les nouvelles technologies, et donc elles produisent, vendent pour gagner un peu d'argent et reproduire, il s'agit en fait d'un cycle « vicieux » où il n y a aucune place pour la planification, l'innovation et les grandes ambitions ; un cycle non productif d'une vraie richesse. De même, élargir sa part du marché, et renforcer ses résultats requièrent toute une planification et des stratégies flexibles pour chaque aspect, notamment la relation avec les clients et le management du changement. Il faut avoir conscience que nous sommes dans un monde où seul le changement est permanent; le changement même des liens sociaux, notamment avec les clients.

A ce niveau, il est important de signaler que $74 \%$ des coopératives étudiées n'ont pas une stratégie dédiée aux clients, une stratégie pour connaitre leurs clients et les segmenter, mesurer leur degré de satisfaction et évaluer leur qualité de service. Ces entités ne sont pas dotées d'une vision claire vis-à-vis de leur clientèle. De ce fait, le contact entre les coopératives et leurs clients est très limité (mal appréciation des exigences et demandes des clients), malgré que nous sommes dans un environnement où les contacts sont devenus de plus en plus faciles à prendre et à maintenir, notamment via les techniques de l'information et de la communication : les réseaux sociaux par exemple.

Les entretiens que nous avons menés, nous ont permis de découvrir que $70 \%$ des coopératives n'ont pas une page web, ni une page sur un réseau social (facebook à titre d'exemple), pour exposer leurs produits, et attirer un nombre important de clients. Encore plus, les coopératives enquêtées ne font pas recours à la technologie dans leur organisation interne, ni pour le suivi de leurs 
ventes ; les produits sont vendus très souvent de manière traditionnelle (à travers des épiceries, des ventes occasionnelles lors des salons d'exposition, les souks, au sein de la coopérative elle-même, pour ce dernier canal la vente dépend des recommandations d'un client à l'autre). Par conséquent, de fortes opportunités ne sont pas saisies, un grand potentiel est mal exploité, et une richesse énorme est gaspillée, le résultat final: des coopératives non compétitives et moins performantes.

Cette situation se traduit clairement sur le terrain. Après presque un demisiècle de la naissance des coopératives au Maroc, leur croissance est très lourde ainsi que leur contribution au PIB national est très timide. Bref, l'amélioration de cet état des choses, suppose le renforcement de la compétitivité des coopératives, ceci n'est possible que par l'adaptation à la révolution technologique et l'intégration du client dans la chaine de production et de commercialisation, étant un vecteur stratégique et une source réelle de la richesse. Réussir la transformation vers « une coopérative digitale/compétitive » requiert l'union des efforts des différentes parties prenantes et la mise en place de nouvelles démarches connectées avec les transformations en cours.

Dans ce contexte, nous devons indiquer l'importance de l'inspiration des expériences internationales en matière du management coopératif. En effet, la mise en place de nouvelles stratégies innovantes et connectées avec les nouvelles transformations des sociétés et respectant les exigences et les attentes des clients contemporains a des répercussions très positives sur la productivité des coopératives dans plusieurs pays et leur contribution au développement socioéconomique, ces stratégies sont axées essentiellement sur ce qui a été appelé par Francis Boyer les « $4 \mathrm{C}$ » à savoir : la confiance, le choix, la coopération, et la convivialité. Grace à ces nouvelles pratiques managériales les coopératives produisent $72 \%$ du blé, $43 \%$ du soja, $39 \%$ du lait, $38 \%$ du coton et $21 \%$ du café au Brésil. En Uruguay, c'est $90 \%$ du lait, $34 \%$ du miel et $60 \%$ du blé national qui sont produits par des coopératives. En Inde, 100000 coopératives laitières collectent chaque jour 16,5 millions de litres de lait de 12 millions d'agriculteurs membres. Concernant la création d'emploi, au Kenya 250000 personnes travaillent dans les coopératives; en Colombie, une coopérative du secteur de la santé occupe la deuxième place des entreprises en ce qui concerne le nombre d'employés. (cité par Ernesto Molina, dans la revue vie économique, volume 3, numéro 4).

\subsection{LA RELATION COOPERATIVES-CLIENTS: LES MESURES DE PERFECTIONNEMENT}

A l'instar des entreprises commerciales, les coopératives doivent avoir conscience que renforcer leur compétitivité et garantir leur survie suppose de mettre le client au centre des leurs intérêts en tant qu'un axe stratégique. Pour 
ce faire, il faut aller au-delà des démarches traditionnelles destinées à fidéliser le client via les techniques simples du marketing, notamment assurer un bon rapport produit/prix (ou qualité/prix). De plus, il n'est même plus suffisant de considérer le client comme un roi, car ses attentes ont déjà dépassé cette considération. Le client d'aujourd'hui, qui est un client averti, de plus en plus exigeant, cherche une relation de nature particulière voire unique avec l'entreprise, il cherche à vivre une expérience extraordinaire. Permettre à ses clients de vivre une telle expérience nécessite le recours à la CRM et ses techniques par la coopérative.

Dans cette perspective, les coopératives au Maroc, sont appelées à repenser leurs démarches et faire évoluer leur organisation interne, pour s'adapter aux grands changements de l'environnement, et assurer une longue vie dans un monde qui ne cesse d'évoluer jour après jour. Il s'agit en fait de changer le management plus que manager le changement.

$\mathrm{Ci}$-dessous, certaines propositions et recommandations :

- La technologie digitale: Au fait, intégrer les nouvelles technologies dans le quotidien de la coopérative est un impératif pour leur développement, vu leur grand potentiel en termes d'innovation, de connaissance des clients, de commercialisation des produits, et d'ouverture sur de nouveaux marchés...Etc. La technologie digitale permet également la création d'un sentiment de proximité et de disponibilité permanente chez les clients.

- Intégrer le client : faire du client une partie active au sein de la coopérative est une stratégie intelligente pour gagner sa confiance. Demander son avis avant, pendant et après la production ou l'offre d'un bien ou un service, est un simple geste qui pourrait lui donner le sentiment d'appartenance à la coopérative. Ce sentiment est un indice de réussite et un facteur pour mieux positionner sur le marché.

- $\quad$ Faire confiance au client : le client de ce jour que l'on peut qualifier de client «contemporain » a besoin de ressentir que la coopérative partage tout avec lui, sans tout partager à propos de lui (ne pas partager ses données personnelles sur les réseaux sociaux par exemple) et prendre en considération ses recommandations et propositions (une composition particulière pour un produit donné, ajouter un goût donné à un produit, les couleurs utilisées sur l'emballage...) des astuces qui peuvent paraître secondaires pour la coopérative, mais qui comptent beaucoup pour le client, et lui donnent l'impression que la coopérative lui fait confiance.

- Mettre en place une stratégie pour fidéliser le client : 20\% des clients rapportent $80 \%$ des revenus, c'est un principe fameux et simple, qui pourrait permettre à la coopérative d'améliorer son rendement. Pour ce faire, il faut être tout proche du client, et renforcer davantage le contact 
avec lui, en se référant aux nouvelles technologies. Ces dernières constituent pour le client une source d'information et d'évaluation des produits ; c'est donc facile d'y procéder pour faire des consultations avant toute décision d'achat. De ce fait, la coopérative doit les exploiter pour satisfaire ses clients et les fidéliser.

- Ventes en ligne: la plupart des coopératives qui négligent le volet technologique dans le circuit des ventes, rencontrent des difficultés de commercialisation, et ratent en effet l'occasion d'améliorer leurs bénéfices. En revanche, les coopératives ont toutes les compétences pour faire du commerce électronique un levier de développement, il faut juste en croire, l'exploiter et faire le premier pas.

- Développement des applications mobile : les mobiles sont devenus entre les mains de tout le monde, une source de l'information par excellence ; pourquoi alors ne pas exploiter cet outil par la coopérative pour accompagner ses clients, notamment via le développement des applications qui rassemblent «la coopérative digitale » et le client digital »; c'est un véritable outil pour fidéliser les clients et créer une relation source d'engagement et de satisfaction, une relation de qualité et durable.

- La formation: veiller à la formation continue des adhérents de la coopérative est un axe important pour diffuser la culture « du client acteur de la réussite ». La négligence du rôle suprême des nouvelles technologies, l'ignorance de leur utilisation et le manque voire l'absence de connaissances sur le marché, le marketing, la communication, la planification...Etc. sont sans doute les conséquences de la négligence du volet formation au sein de la coopérative, par la coopérative elle-même mais aussi par les autorités concernées (ODCO par exemple) tout au long de la durée d'activité de la coopérative, et surtout à la première phase de la constitution. De ce fait, donner à la formation l'importance qu'elle mérite ne peut qu'enrichir le parcours de la coopérative en termes de réalisations positives.

- La CRM: l'application réussie de la CRM comme stratégie de fidélisation des clients, demande pour la coopérative de réussir l'ensemble des points précédents, car elle requiert de réunir un ensemble de facteurs liés à la coopérative (l'entreprise), son organisation, ses adhérents, ses stratégies, son réseau...etc. sans doute, mettre en place les techniques de la CRM permettra à la coopérative de renforcer sa compétitivité et sa performance, mais il ne faut pas oublier que cela suppose la maitrise de la conduite des changements, vu que l'intégration de la CRM engendre des transformations organisationnelles importantes, et demande le développement de 
nouvelles compétences et des méthodes de travail (Fabienne AUBER, 2011). Bref, la réussite à l'intégration de la CRM reflète la maturité managériale de la coopérative.

Pour clôturer ce point, il est indispensable d'indiquer que quelques soient les propositions citées ou les idées recommandées, aucun résultat ne sera atteint sans avoir unifié les efforts des différentes parties prenantes.

\section{CONCLUSION:}

La relation avec les clients constitue l'une des préoccupations majeures de toutes entreprises, dans tous les secteurs. Cette relation a connu une forte évolution les dernières années. Elle se traduit par l'importance grandissante de la technologie digitale, et sa domination sur la vie de l'entreprise. Les coopératives n'échappent pas à cette réalité. De ce fait, elles doivent prendre en compte que leurs clients ont plus de pouvoir et d'influence sur leur compétitivité ; ces clients qui sont aussi dirigés par les nouvelles technologies s'attendent à ce que les services/produits présentés par la coopérative soient plus sophistiqués et représentent le progrès qu'ils constatent dans leur quotidien.

Dans ce sens, la compétitivité de la coopérative est parfaitement conditionnée par la fidélisation des clients voire « l'expérience client », cette dernière suppose de nouveaux ingrédients dans la stratégie de la coopérative, notamment le digital, étant la pièce angulaire pour la survie de toute organisation.

Bref, nous vivons dans un monde où la révolution technologique a tout bouleversé : le comportement des consommateurs et l'organisation interne des entreprises y compris les coopératives. S'adapter à ce nouveau contexte est une condition primordiale pour la compétitivité et la survie. De plus, tout porte à croire que nous ne sommes qu'au début du vrai changement du monde, et qu'il continuera à évoluer d'un rythme plus fort dans les prochaines années.

Pour conclure, ce travail ne prétend pas porter une réponse exhaustive aux différents aspects que le thème soulève, mais il représente une tentative d'explication de la relation des coopératives avec leurs clients au Maroc. Il requiert des recherches supplémentaires et approfondies, ainsi que des études empiriques pour faire de la GRC un levier de développement des coopératives, et proposer des modèles permettant l'évaluation et la mesure de l'impact réel de cette relation sur la compétitivité des coopératives. 
Annexe 1 : Guide d'entretien

Question d'ouverture :

\begin{tabular}{|c|c|}
\hline \hline \multicolumn{2}{|c|}{ Identification de la coopérative } \\
\hline Coopérative & \\
\hline Activité & \\
\hline Adresse & \\
\hline Nombre d'adhérents & \\
\hline
\end{tabular}

\section{Question d'introduction :}

Pouvez-vous me donner une idée plus précise sur votre coopérative (la procédure, la date de création, le financement, les difficultés, les ambitions ?

\section{Question de transition :}

Concernant vos ambitions, j'imagine que vous souhaitez élargir votre marché, comment pensez-vous de le faire?

\section{Questions clés :}

- Comment produisez-vous essentiellement, (méthodes traditionnelles, ou modernes)

- Si modernes, quelles techniques, ou types de machines vous utilisez ? comment vous les avez obtenus (fonds propres, dons...)?

- Vous produisez sur commande, ou bien vous produisez et puis vous cherchez des acheteurs?

- Quel type de clients envisagez-vous?

- Comment vous faites connaitre vos produits ?

- Faites-vous de la publicité ? (Si oui par quel moyen ?)

- Avez-vous un ordinateur au sein de votre coopérative? avez-vous accès à l'internet?

- Avez-vous une page sur l'un des réseaux sociaux (site web, Facebook, applications mobiles...?

- Réellement, quelle est votre relation avec les nouvelles technologies ?

- A votre avis, est-il possible de renforcer votre présence sur le marché sans recours aux nouvelles technologies?

- Comment qualifier vous votre relation avec vos clients?

- Vos clients sont-ils satisfaits ? comment le saviez-vous ?

- Est-ce que vous pensez que la manière dont vous produisez et les conditions où vous travaillez vous permettront de fidéliser vos clients et d'attirer une nouvelle clientèle ?

- Pourquoi ? qu'est-ce qu'il manque réellement? 


\section{Question de fermeture :}

A votre avis, de quoi vous avez besoin aujourd'hui pour fidéliser vos clients, assurer votre survie et devenir compétitifs?

\section{References:}

1. Paul Bouvier Patron. (2011), «Economie et management de l'entreprise innovante : réseau, territoire et développement durable », L'Harmattan.

2. E. Bayle, J.C. Dupuis. (2012), «management des entreprises de l'économie sociale et solidaire », De Boeck Supérieur.

3. Christine Marson, (2008). «Réussir le changement individuel et collectif », Manager RH.

4. Sophie Dubuison Quellie. (2009), «La communication engagée », Contester.

5. Bruno Joly. (2009), « La communication », De Boeck Supérieur.

6. Alain Maes. (2012), «Le management intégrateur: fondements, méthodes et applications », De Boeck Supérieur.

7. Roger Mehl. (1984), « Essai sur la fidélité », Hors collection.

8. Martens, S et al (2010), « la gestion des entreprises sociales », Liège, Edipro.

9. Enjolras B. (1992), « Services de proximité dans l'action sociale, l'apport de la théorie du choix institutionnel », Recma, n 45-46.

10. N. Audigier, M.A De Gail et L. Derien (2016), «Optimiser l'évaluation du client en ligne », La revue des sciences de gestion, $\mathrm{N}^{\circ}$ 278/279 www.cairn.info.

11. L. Body, C. Tallec (2015), “L'expérience client”, EYROLLES

12. S. Ahrouch (2015), « les coopératives au Maroc: enjeux et évolutions », RECMA, $\mathrm{N}^{\circ} 322$.

13. MEDEF, (2015), «Guide de bonnes pratiques: L'utilisation du numérique dans les relations clients », www.medef.com

14. INCR, (2014), «L'excellence de la relation par les compétences », Www.eurogroupconsulting.com

15. Themeco (2016), « la transformation digitale des entreprises », www.themeco.fr

16. Davis. J. (1992), « Ethics and Environmental Marketing», Journal of Business Ethics, Vol.11, N², p 65-67.

17. "Etude de la coopérative digitale", (en ligne), disponible sur : https://www.sommetinter.coop (consulté le 26/02/2018).

18. Fabien (2016), « cinq raisons qui rendent la mesure de la satisfaction client indispensable $»$ (en ligne), disponible sur : https://business.critizr.com (consulté le 02/02/2018). 
19. Thomas Coeffé (2017) chiffres internet-2017 [en ligne] Disponible sur : https://www.blogdumoderateur.com (consulté le 03/03/2017)

20. ODCO, (2015), « Annuaire statistique des coopératives et leurs unions au Maroc pour l'année 2015 ».

21. Ed Peelen, F. Jallat et E. Stevens, (2017), « Gestion de la relation client : Total relatinship management, big data et marketing mobile ", Pearson.

22. P. Alard, et P.A Guggémos (2004), "CRM- les clés de la réussite " Editions d'Organisation.

23. S. Brown, (2006), «CRM : la gestion de la relation client », village mondial.

24. R. Lef2bure et C. Venturi, (2005) «Gestion de la relation client», EYROLLES.

25. Zott.C. et Amit.R. (2008), « the fit between product market strategy and business model: implications for firm performance», Strategic Management Journal, Vol.29, p: 5-15.

26. Wood.D.J. (1991), «Corporate Social Performance Revisited», Academy of Management Journal, Vol.16, pp. 316-327.

27. Draperi J.F(2003), « La coopération face aux nouvelles attentes de la société », in Touzard $\mathrm{J}$. 